\title{
Concurrence of prenatally diagnosed Cantrell pentalogy and short and tight umbilical cord: $A$ possible case of hexalogy
}

\author{
Abdussamet Batur ${ }^{1, *}$, Cemil Goya $^{2}$, Alpaslan Yavuz $^{1}$, Cihad Hamidi ${ }^{2}$, Mehmet Guli Cetincakmak ${ }^{2}$ \\ ${ }^{1}$ Department of Radiology, Yuzuncu Yil University Dursun Odabas Medical Center, Van \\ ${ }^{2}$ Department of Radiology, Dicle University School of Medicine, Diyarbakir
}

\begin{abstract}
Cantrell pentalogy is a rather rare syndrome characterised by complete or partial ectopia cordis; together with omphalocele, congenital heart diseases, and defects in the pericardium, lower sternum and anterior diaphragm. In this article, our aim is to present the ultrasonographic evidence of the concurrence of a short umbilical cord and the Cantrell syndrome, which we believe will contribute to the literature.
\end{abstract}

Key Words: Cantrell pentalogy, ectopia cordis, umbilical cord

\section{Introduction}

Cantrell pentalogy (PC) is a syndrome characterised by a complete or partial ectopia cordis; together with omphalocele, congenital heart diseases, and defects in the pericardium, lower sternum and anterior diaphragm. The syndrome has first been reported in 1958 by Cantrell, Haller and Ravitch in a group of 5 patients (1). It is a rare syndrome observed in $1 / 65.000-1 / 200.000$ births. The rare concurrence of Cantrell pentalogy and a short umbilical cord, which we have detected through ultrasound at an early phase of the gestation, is a new finding undefined by Cantrell et al. (1) Thus, we would like to present our case since we are of the opinion that it may contribute to the literature with a new concept (Cantrell hexalogy?).

\section{Case report}

A 33-year old patient in her first pregnancy, whose last menstrual period date was unknown, was sent to our clinic by the Gynaecology and Obstetrics Department with a suspected fetal anomaly. No intra-familial marriage was concerned and there was nothing remarkable in the patient's history. In her repeated ultrasonographic examinations first at the Gynaecology and Obstetrics, and later at the
Radiology Departments, a single live intrauterine pregnancy at 11 weeks and 3 days was observed. A large defect was present at the frontal abdominal wall of the fetus and some of the intra-abdominal organs and part of the heart were located extracorporeally at the level of this defect (Figure 1,2). The defect was surrounded with a membrane and the umbilical cord was observed inferiorly of the pouch. The umbilical cord was short, straight and tight (Figure 3). The view of the uterus was consistent with contraction. The umbilical cord was observed to directly join the right atrium. The diameter of the fetal thorax was small (hypoplasia). A cystic hygroma was observed in the fetus. The Doppler echographic examination revealed a single-cavity heart without a septum. Based on the fetal omphalocele and ectopia cordis evidence, the patient was prediagnosed with Cantrell pentalogy and a short umbilical cord.

In the detailed examination of the patient in an advanced diagnostic centre, these findings were confirmed. With the request and approval of the patient and her relatives, a medical evacuation was performed. Samples were taken from the aborted material for the genetic assessment of the fetus. The genetic assessment using the conventional cytogenetic (long-term culture) method revealed a 46, XY normal karyotype. 


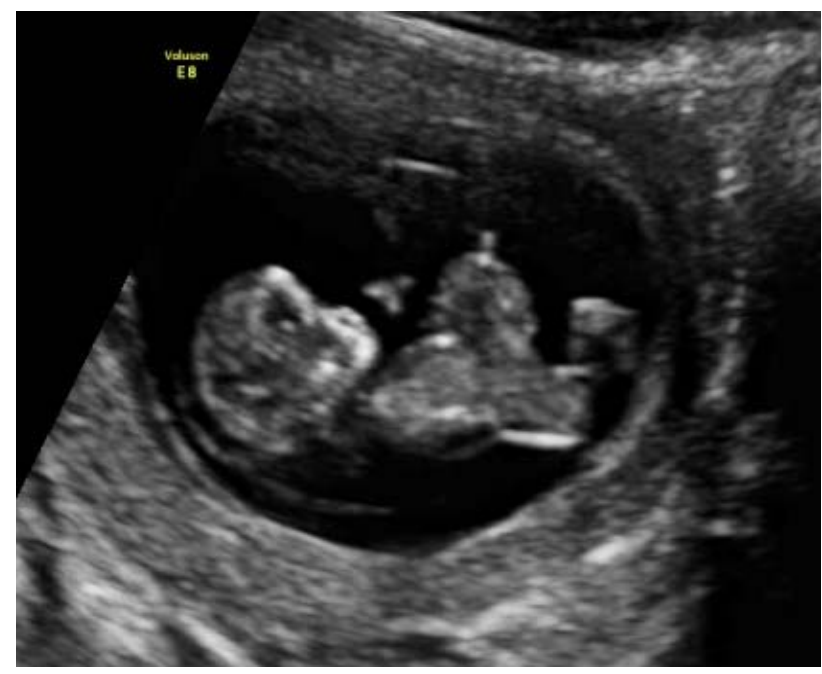

Fig. 1. Ultrasonographic view of the fetal omphalocele and the ectopically located heart (ectopia cordis) inside. The short-tight and straight umbilical cord is visible.

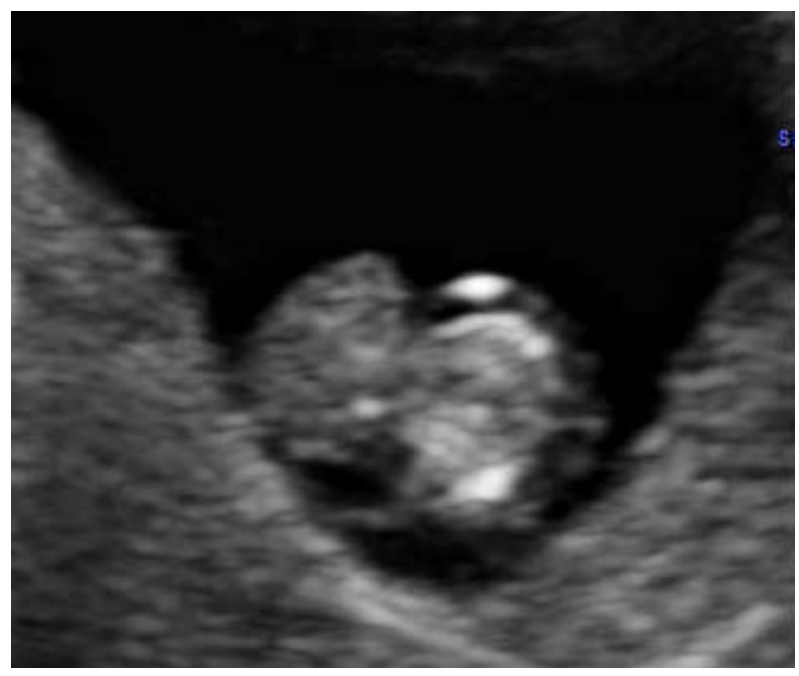

Fig. 2. Thoracoabdominal defect and the ectopic heart with the omphalocele.

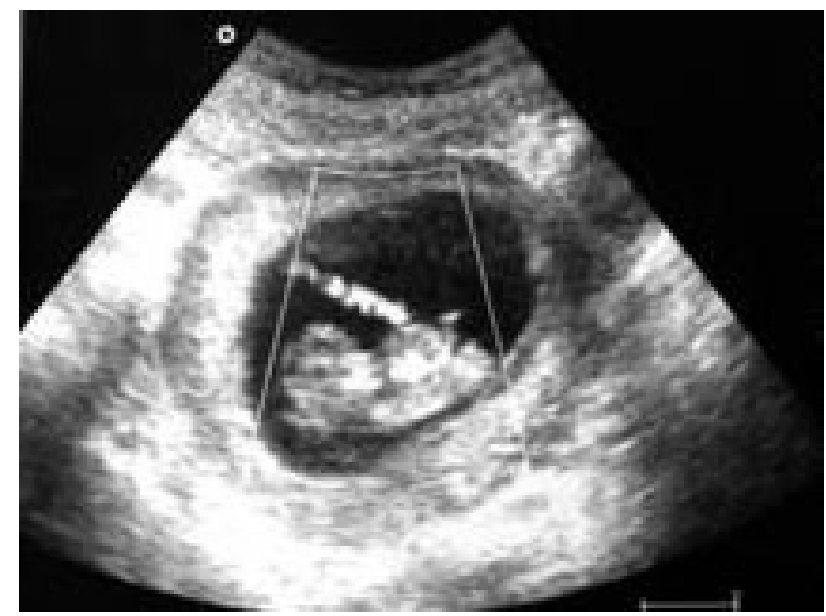

Fig. 3. Short, tight and straight UC; shorter than the CRL: UC directly joins the right atrium (ductus venosus anomaly).

\section{Discussion}

Cantrell pentalogy is also known as the CantrellHaller-Ravitch syndrome. The Cantrell syndrome is thought to occur due to a defect in the formation of the segmental mesoderm 14-18 days after the conception (1). As a result, the transverse septum is not developed in the diaphragm and the ventromedial migration of the mesodermal folds into the upper abdomen is disturbed.

The problem is thought to occur during the $14^{\text {th }}$ $15^{\text {th }}$ gestational days when the splanchnic and parietal mesoderms are not differentiated yet. While the effect on the splanchnic mesoderm causes the defects in the heart and the pericardium, the influence on the parietal mesoderm brings about the defects on the abdominal wall and the diaphragm (2).

Since the first case reported by Cantrell, only a limited number of cases has been reported and the frequency of complete pentalogies is even lower (3). While there may be severe forms of the pentalogy, less severe forms may also be observed. In this syndrome called the Cantrell pentalogy, not every patient may show all the 5 components of the syndrome. In 1972, Toyoma et al. (4) have classified the cases in 3 groups according to the level of expression of the pentalogy. According to this classification, Type 1 shows all the 5 diagnostic defects. Type 2 has 4 or 5 of the defects with an estimated diagnosis. Type 3 shows varying levels of the defects in different combinations and 
incomplete expressions $(5,6)$. In our case, our patient had all the findings described by Cantrell.

Some of the congenital anomalies observed together with the Cantrell syndrome are the cloacal exstrophy, genitourinary and vertebral anomalies, occipital encephalocele, horseshoe kidney, bilateral clubfeet, spina bifida, hydrocephaly, abnormal ears, trisomy 18, exencephaly, pulmonary hypoplasia, cystic hygroma, craniorachischisis, and cleft lip and palate. The most commonly observed congenital cardiac disorders in these patients are ventricular septal defects, followed by atrial septal defects, left ventricular diverticulitis, tetralogy of Fallot, double outlet right ventricle and pulmonary stenosis (7).

Since the development of the PC and umbilical vein formation go hand in hand during the embryonic stage, Brochut et al. (8) have suggested that their pathologies may also be interconnected. In terms of the umbilical pathologies, they have detected a single umbilical artery, short umbilical artery and atypical coiling patterns in four patients. Since the PC and the pathologies of the umbilical cord are in the same malformation group, they have claimed that they may change the early fetoplacental blood flow and blood vessel structure. The investigators have also emphasized that further studies are needed in order to determine if the anomalies of the umbilical cord are a part of this syndrome (8). The fetus in our case also had a short, straight and tight umbilical cord and the cord was directly joining the right atrium. These findings support the thesis that the case may be a hexalogy.

In conclusion, in patients where a Cantrell pentalogy is detected, the umbilical cord must be carefully examined and any pathology related to the umbilical cord should not be overlooked.
Investigated from this point of view, we are of the opinion that the ratio of the umbilical cord pathologies will increase in the patients where the pentalogy is diagnosed, which may lead to a change in the name of the syndrome as the Centrall hexalogy in the future.

\section{References}

1. Cantrell JR, Haller JA, Ravitch MM. A syndrome of congenital defects involving the abdominal wall, sternum, diaphragm, pericardium, and heart. Surg Gynecol Obstet 1958; 107: 602-614.

2. Ghidini A, Sirtori M, Romero R, Hobbins JC. Prenatal diagnosis of pentalogy of Cantrell. J Ultrasound Med 1988; 7: 567-572.

3. Correa-Rivas MS, Matos-Llovet I, García-Fragoso L. Pentalogy of Cantrell: a case report with pathologic findings. Pediatr Dev Pathol 2004; 7 : 649-652.

4. Toyama WM. Combined congenital defects of the anterior abdominal wall, sternum, diaphragm, pericardium and heart: a case report and review of the syndrome. Pediatrics 1972; 50: 778-792.

5. Van Hoorn JHL, Moonen RMJ, Huysentruyt CJR, et al. Pentalogy of Cantrell: two patients and a review to determine prognostic factors for optimal approach. Eur J Pediatr 2008; 167: 29-35.

6. Sepulveda W, Weiner E, Bower S, et al. Ectopia cordis in a triploid fetus: first trimester diagnosis using transvaginal color Doppler ultrasonography and chorionic villous sampling. J Clin Ultrasonogr 1994; 22: 573-575.

7. van Hoom JH, Moonen RM, Huysentruyt CJ, et al. Pentalogy of Cantrell: two patients and a review to determine prognostic factors for optimal approach. Eur J Pediatr 2008; 167: 29-35.

8. Brochut AC, Baumann MU, Kuhn A, et al. Pentalogy or hexalogy of Cantrell? Pediatr Dev Pathol 2011; 14: 396-401. 